\title{
Nefritis como debut lúpico en el embarazo. Reporte de dos casos y revisión de la literatura
}

\section{Nephritis as a lupic debut in pregnancy. Report of two cases and review of the literature}

\author{
Isabel Hinojal-Toscano* y Mónica Marín-Cid \\ Unidad de Gestión Clínica de Medicina Fetal, Genética y Reproducción, Hospital Universitario Virgen del Rocío, Sevilla, España
}

\section{Resumen}

\begin{abstract}
Antecedentes: El lupus eritematoso sistémico es una enfermedad autoinmunitaria multisistémica que afecta principalmente a mujeres en edad fértil. La nefritis lúpica es la manifestación clínica más frecuente durante la gestación y constituye un factor de riesgo para la pérdida del embarazo, en especial en pacientes con insuficiencia renal. Además, presenta mayor riesgo de pérdida fetal, restricción del crecimiento intrauterino e hipertensión. Casos clínicos: El primer caso se trata de una gestante de 28 + 2 semanas con daño renal grave y anticuerpos anti-DNA en título elevado. En el segundo caso destaca el debut con sintomatología de dificultad respiratoria y edemas como manifestaciones clínicas de nefropatía lúpica tipo $\mathrm{V}$ a las 23 semanas de gestación. En ambas pacientes destaca la prematuridad como complicación perinatal, así como el crecimiento intrauterino retardado en el primer caso. Por último, se describen los resultados clínico-analíticos tras el inicio terapéutico específico en ambos casos. Conclusiones: El diagnóstico diferencial del debut de nefritis lúpica durante la gestación continúa siendo un desafío, a pesar de los avances en cuanto a marcadores angiogénicos. La valoración clínica continúa siendo la piedra angular de este proceso diagnóstico y de sus implicaciones en cuanto a complicaciones del embarazo actuales y futuras.
\end{abstract}

Palabras clave: Nefritis lúpica en gestante. Lupus eritematoso sistémico. Complicaciones de lupus en gestación. Manejo de nefritis lúpica.

\section{Abstract}

Background: Systemic lupus erythematosus is a multisystem autoimmune disease that mainly affects women of childbearing age. Lupus nephritis represents the most frequent clinical manifestation in pregnancy, constituting a risk factor for pregnancy loss, especially in patients with kidney damage. It also has a higher risk of fetal loss, intrauterine growth restriction, and gestational hypertension. Clinical cases: The first case is a $28+2$-week pregnant woman with severe kidney damage and high-titles anti-DNA antibodies. In the second case, we highlight the debut with symptoms of respiratory distress and edema as clinical manifestations of type $V$ lupus nephropathy in a 23-week gestation. In both cases, prematurity stands out as a perinatal complication, as well as delayed intrauterine growth in the former. Finally, the clinical-analytical results are described, after the specific therapeutic initiation in both cases. Conclusions: The differential diagnosis of the onset of lupus nephritis during pregnancy continues to be a challenge, despite the advances in angiogenic markers; clinical assessment continues to be the cornerstone of this diagnostic process and its implications for current and future pregnancy complications.

Keywords: Lupus nephritis gestation. Systemic lupus erythematosus. Lupus complications in pregnancy. Lupus nephritis management.

Disponible en internet: 07-02-2022 Rev Chil Obstet Ginecol. 2021;86(6):545-553

www.rechog.com 


\section{Antecedentes}

El lupus eritematoso sistémico (LES) es una enfermedad autoinmunitaria multisistémica que afecta principalmente a mujeres en edad fértil. Las mujeres con LES tienen una tasa de fertilidad normal, haciendo del embarazo un tema de interés frecuente en estas pacientes $^{1,2}$. Según las distintas fuentes consultadas, el LES tiene una prevalencia estimada de 20 a 150 casos por 100.000 y una incidencia de 1 a 25 casos por 100.000 personas/año, y de 1/1000 mujeres de entre 15 y 45 años ${ }^{1,2}$.

La incidencia de LES se estima en 9 por 10.000 habitantes, afectando principalmente a mujeres de edad fértil, con una razón mujer/hombre de 10/1, lo que traduce una relación entre las hormonas sexuales y la etiología y la fisiopatología de la autoinmunidad ${ }^{2,3}$.

El embarazo y el LES se ven afectados de manera recíproca ${ }^{2}$. El embarazo puede aumentar la actividad del LES y tener efectos adversos a corto o largo plazo sobre la función renal ${ }^{1,3}$. La nefritis lúpica es la tercera forma de presentación clínica del lupus fuera del embarazo, pero en la gestación representa la manifestación clínica más frecuente ${ }^{4}$. La nefritis lúpica es un factor de riesgo para la pérdida del embarazo, especialmente en pacientes con insuficiencia renal ${ }^{5}$. Además, presenta mayor riesgo de pérdida fetal, crecimiento intrauterino restringido $(\mathrm{CIR})$ e hipertensión gestacional, en un $30-50 \%$, incluso en pacientes con nefritis lúpica con LES no activo $0^{6,7}$.

Se considera que el mejor momento para la concepción es tras 6 meses de estabilidad clínica, ya que los resultados adversos del embarazo están relacionados con la nefritis activa ${ }^{6}$. Esto puede deberse a una microcirculación comprometida en los vasos uteroplacentarios de las pacientes con LES con hipertensión arterial (HTA) y nefritis lúpica ${ }^{7}$.

Un ensayo clínico publicado por Magee et al. ${ }^{8}$ en 2015 determinó que un control más estricto de las cifras tensionales se asociaba a una disminución del riesgo de desarrollar HTA grave (> 160/110 $\mathrm{mmHg}$ ), del $40,6 \%$ al $27,5 \%(p<0,001)$.

Todas las mujeres con glomerulonefritis son candidatas para estrategias preventivas. Las dosis bajas de ácido acetilsalicílico se consideran en la actualidad como parte de la atención estándar, ya que se han asociado con una reducción significativa de la preeclampsia (riesgo relativo [RR]: 0,47; intervalo de confianza del 95\% [IC95\%]: 0,34-0,65) y el CIR (RR: 0,44; IC95\%: 0,30-0,65 $)^{9,10}$. Además, resultó en una reducción significativa de preeclampsia grave y de parto prematuro. Del mismo modo, se ha demostrado que la suplementación con calcio reduce la incidencia de preeclampsia de manera más pronunciada en aquellas mujeres con bajo consumo de calcio (RR: 0,48; IC95\%: $0,33-0,69)^{11,12}$.

El diagnóstico de nefritis lúpica en una gestante sana supone un desafío importante, ya que suele plantearse como un diagnóstico de segunda o tercera línea, una vez descartadas otras patologías más frecuentes. El manejo interdisciplinario ayuda a establecer un diagnóstico más temprano y facilita un inicio terapéutico precoz.

Presentamos dos casos de debut de nefritis lúpica atendidos en nuestro hospital y el diagnóstico diferencial con otras afecciones, como la preeclampsia, el síndrome hemolítico urémico y otros tipos de glomerulonefritis. Resaltamos la dificultad diagnóstica añadida al suceder el primer caso clínico durante la pandemia mundial de COVID-19. Además, realizamos una revisión bibliográfica sobre su manejo y complicaciones.

\section{Caso 1: nefritis lúpica. Debut en la semana 28 de gestación}

Mujer de 30 años, gestante de $28+2$ semanas de gestación (SG), que acudió por dolor abdominal irradiado a ambas fosas renales, asociado a presión arterial de $144 / 80 \mathrm{mmHg}$. Antecedentes: dos partos anteriores, siendo el segundo en la semana 34 con rotura prematura de membranas en la semana 27. Gestación actual de escaso control.

Ingresó con diagnóstico de HTA a estudiar y amenaza de parto pretérmino, por lo que se comenzó tratamiento tocolítico con atosibán, neuroprotección con sulfato magnésico y maduración pulmonar fetal a su llegada.

En las pruebas complementarias realizadas durante su ingreso se objetiva: anemia grave con hemoglobina de 6,1 g/dl junto con discreta linfocitopenia; lesión renal aguda de grado II con creatinina de 1,6 mg/dl y urea de $66 \mathrm{mg} / \mathrm{dl}$. Frotis en sangre periférica normal. Análisis elemental de orina patológico: leucocituria, proteinuria y microhematuria; proteinuria de 24 horas de 5 . Prueba de reacción em cadena de la polimerasa para COVID19 negativa.

Se realizaron las siguientes pruebas de imagen:

- Ecografía obstétrica: cervicometría de $28 \mathrm{~mm}$.

- Ecografía transabdominal: cefálica, latido fetal y movimientos fetales positivos, placenta normoinserta, líquido amniótico normal. Peso fetal estimado de $903 \mathrm{~g}$. Índice de pulsatilidad de la arteria umbilical 1,1 (percentil 49). Índice de pulsatilidad de la arteria 
Tabla 1. Diagnóstico diferencial

\begin{tabular}{|c|c|c|c|c|c|c|c|}
\hline & $\begin{array}{l}\text { Tipo de } \\
\text { insuficiencia } \\
\text { renal }\end{array}$ & $\begin{array}{l}\text { Lactato } \\
\text { deshidrogenasa }\end{array}$ & Plaquetas & Transaminasas & Hematuria & $\begin{array}{l}\text { Presión } \\
\text { arterial }\end{array}$ & Otros datos \\
\hline $\begin{array}{l}\text { Preeclampsia } \\
\text { con criterios de } \\
\text { gravedad }\end{array}$ & $\begin{array}{l}\text { Síndrome } \\
\text { nefrótico }\end{array}$ & $\begin{array}{l}\text { Normal o } \\
\text { aumentada }\end{array}$ & $\begin{array}{l}\text { Normales o } \\
\text { disminuidas }\end{array}$ & $\begin{array}{l}\text { Normales } 0 \\
\text { elevadas }\end{array}$ & No & Elevada & $\begin{array}{l}\text { Arterias uterinas } \\
\text { patológicas }\end{array}$ \\
\hline $\begin{array}{l}\text { Síndrome } \\
\text { hemolítico } \\
\text { urémico }\end{array}$ & $\begin{array}{l}\text { Síndrome } \\
\text { nefrítico }\end{array}$ & Aumentada & Disminuidas & $\begin{array}{l}\text { Normales } 0 \\
\text { elevadas }\end{array}$ & Sí & $\begin{array}{l}\text { Normal } \\
0 \\
\text { elevada }\end{array}$ & $\begin{array}{l}\text { Complemento } \\
\text { normal }\end{array}$ \\
\hline $\begin{array}{l}\text { Glomerulonefritis } \\
\lg A\end{array}$ & $\begin{array}{l}\text { Síndrome } \\
\text { nefrítico }\end{array}$ & Normal & Normales & Normales & Sí & Elevada & $\begin{array}{l}\text { Amigdalitis de } \\
\text { repetición }\end{array}$ \\
\hline $\begin{array}{l}\text { Glomerulonefritis } \\
\text { secundarias } \\
\text { (lupus o } \\
\text { infección ) }\end{array}$ & $\begin{array}{l}\text { Síndrome } \\
\text { nefrítico }\end{array}$ & Normal & Normales & Normales & Posible & Elevada & Hipocomplementemia \\
\hline $\begin{array}{l}\text { Nefropatía } \\
\text { diabética }\end{array}$ & $\begin{array}{l}\text { Síndrome } \\
\text { nefrótico }\end{array}$ & Normal & Normales & Normales & No & Normal & Hiperglucemia \\
\hline
\end{tabular}

cerebral media 1,46 (percentil 5). Índice cerebro-placentario 1,32 (percentil 6). Índice de pulsatilidad de las arterias uterinas 1,71 (superior al percentil 99). CIR tipo I. Ecografía morfológica sin hallazgos significativos.

- Ecografía abdominal: hiperrefringencia renal, líquido perirrenal, derrame pleural.

En este momento se planteó el diagnóstico diferencial entre preeclampsia con criterio de gravedad, síndrome hemolítico urémico o glomerulonefritis IgA (Tabla 1).

Al quinto día de ingreso, el estudio de anemia reportó haptoglobina normal y Coombs directo positivo. Estudio inmunológico: hipocomplementemia C1q, C3 y C4. Títulos de anticuerpos antinucleares (ANA) elevados, anti-DNA > $600 \mathrm{Ud}$, y antiantígeno $\mathrm{A}$ (anti- SSA/Ro) y antiantígeno $B$ (anti-SSB/LA) positivos.

Se diagnosticó debut de nefritis lúpica de probable tipo IV y, como opción menos probable, exacerbación de nefropatía lúpica no diagnosticada. Comenzó tratamiento con hidroxicloroquina, $200 \mathrm{mg}$ cada $12 \mathrm{~h}, \mathrm{y}$ corticoterapia con metilprednisolona en bolo de $250 \mathrm{mg}$ durante 3 días, seguidos de prednisona $50 \mathrm{mg}$ cada $24 \mathrm{~h}$ tras pruebas de alergia negativas.

Durante el ingreso se produjo un deterioro clínico analítico, con aumento de edemas, crepitantes en base pulmonar, elevación de la presión arterial a 160/90 mmHg, acidosis metabólica, hiperpotasemia y fallo renal (creatinina 2,14, filtrado glomerular $22,98 \mathrm{ml} / \mathrm{min}$ y diuresis $800 \mathrm{ml}$ ), por lo que se decidió iniciar inmunosupresión con azatioprina a dosis de
Tabla 2. Evolución de los parámetros analíticos del caso

\begin{tabular}{|c|c|c|c|c|}
\hline & $\begin{array}{c}\text { Al } \\
\text { ingreso }\end{array}$ & $\begin{array}{c}\text { Tras } \\
\text { inicio de } \\
\text { corticoterapia }\end{array}$ & $\begin{array}{c}\text { Día de } \\
\text { cesárea }\end{array}$ & $\begin{array}{c}\text { Tras } \\
\text { cesárea }\end{array}$ \\
\hline $\begin{array}{c}\text { Creatinina } \\
\text { (mg/dl) }\end{array}$ & 1,6 & 1,46 & 2,14 & 1,99 \\
$\begin{array}{c}\text { Hemoglobina } \\
\text { (g/dl) }\end{array}$ & 6,1 & 8,9 & 8,4 & 6,6 \\
$\begin{array}{c}\text { Urea } \\
\text { (mg/dl) }\end{array}$ & 87 & 84 & 110 & 104 \\
\hline $\begin{array}{c}\text { Potasio } \\
\text { (mEq/l) }\end{array}$ & 5,6 & 6,1 & 5,1 & 6,4 \\
\hline
\end{tabular}

$1,5 \mathrm{mg} / \mathrm{kg} / \mathrm{día}$ (Fig. 1). En la semana 30 + 2 se decidió la finalización de la gestación por interés materno. Se indicó cesárea por presentación inestable. Nació un varón vivo de $903 \mathrm{~g}$ que evolucionó sin complicaciones de la prematuridad ni datos de lupus neonatal.

Tras la realización de la cesárea se indicó transfusión por cifras de hemoglobina de 6,1 g/dl e inició tratamiento con ciclofosfamida intravenosa. La paciente permanece estable a los 7 meses desde la cesárea.

\section{Caso 2: nefritis lúpica. Debut en la 23 + 4 semana de gestación}

Mujer de 26 años, gestante con 23 + 4 SG, que consultó por edemas y dificultad respiratoria. Inició clínica de edemas 3 semanas previas al ingreso, sin otra 
Rev Chil Obstet Ginecol. 2021;86(6)

Tabla 3. Comparación de ambos casos clínicos

\begin{tabular}{|c|c|c|c|}
\hline & \multirow[t]{2}{*}{ Tipo de afectación renal } & Gestante de 28 SG & Gestante de 23 SG \\
\hline & & Síndrome nefrítico & Síndrome nefrótico \\
\hline \multirow[t]{6}{*}{ Datos analíticos al ingreso } & Creatinina & $1,6-2$ & $0,69-0,72$ \\
\hline & Hemoglobina (g/dl) & 6,1 & 7,8 \\
\hline & Complemento & Disminuido & Disminuido \\
\hline & ANA & +++ & +++ \\
\hline & Otros autoanticuerpos & Anti-Ro y anti-LA + & Anti-ENA y anti-RNP \\
\hline & Citopenias (líneas) & 2 líneas & 1 línea \\
\hline \multirow[t]{2}{*}{ Datos obstétricos } & CIR I & Sí & Sí \\
\hline & IP arteria uterina & Patológica & Normal \\
\hline \multirow[t]{3}{*}{ Datos clínicos } & Presión arterial & Elevadas & Normales \\
\hline & Edemas & + & +++ \\
\hline & Hematuria & +++ & + \\
\hline \multirow[t]{5}{*}{ Tratamiento } & Inicio hidroxicloroquina & 7. ${ }^{\circ}$ día & $12 .^{\circ}$ día \\
\hline & Inicio corticoterapia & $10 .^{\circ}$ día & 9. ${ }^{\circ}$ día \\
\hline & Inicio azatioprina & $11 .^{\circ}$ día & 9. día \\
\hline & Inicio ciclofosfamida & Tras parto $14 .^{\circ}$ día & En semana $27+4$ \\
\hline & Otros tratamientos & Labetalol & Albúmina+diuréticos \\
\hline Debut vs. exacerbación & Datos clínicos previos & $\begin{array}{l}\text { Elementales de orina } \\
\text { patológicos desde hace } \\
13 \text { años }\end{array}$ & ANA+2012 hacia 3 años \\
\hline \multirow[t]{2}{*}{ Evolución } & Clínica & $\begin{array}{l}\text { Desfavorable } \\
\text { Uremia sintomática }\end{array}$ & Favorable (disminución de edemas) \\
\hline & Analítica & Acidosis metabólica & Hipoalbuminemia \\
\hline \multirow[t]{5}{*}{ Datos parto } & Motivo de terminación & Deterioro materno & $\begin{array}{l}\text { Rotura prematura de membranas en } \\
\text { semana } 34+2\end{array}$ \\
\hline & Semanas parto & $30+2$ & $34+3$ \\
\hline & Vía del parto & $\begin{array}{l}\text { Cesárea (presentación } \\
\text { inestable) }\end{array}$ & Vaginal no instrumental \\
\hline & Peso al nacer & $903 \mathrm{~g}$ & $1598 \mathrm{~g}$ \\
\hline & Apgar al nacimiento & $6 / 8 / 10$ & $10 / 10$ \\
\hline \multirow[t]{2}{*}{ Evolución del recién nacido } & $\begin{array}{l}\text { Complicaciones de } \\
\text { prematuridad }\end{array}$ & No & No \\
\hline & Lupus neonatal & No & No \\
\hline
\end{tabular}

ANA: anticuerpos antinucleares; Anti-ENA: anticuerpos contra antígenos nucleares extraíbles; Anti-LA: anticuerpos anti-síndrome de Sjšgren B; Anti-RNP: anticuerpos anti-ribonucleoproteína; Anti-Ro: anticuerpos anti-síndrome de Sjšgren A; CIR: crecimiento intrauterino restringido; IP: índice de pulsatilidad.

sintomatología asociada en ese momento. Antecedentes: enfermedad de Cushing con microadenoma productor de corticotropina intervenido 2 años antes y cesárea por falta de progresión del parto.
En las pruebas complementarias realizadas durante ingreso para estudio de nefropatía se encuentra: función renal normal con creatinina $0,69 \mathrm{mg} / \mathrm{dl}$ y proteinuria de 24 horas de $11,5 \mathrm{~g}$, sin alteraciones urinarias 
salvo proteinuria, con hipoalbuminemia de $1,7 \mathrm{~g} / \mathrm{dl}$ y descenso de la gammaglobulina sérica.

La ecografía obstétrica halló CIR de tipo I, con estudio Doppler normal tanto fetal como uterino.

En el resto del estudio, hemograma sin linfocitopenia, pero con anemia grave (hemoglobina $7,8 \mathrm{~g} / \mathrm{dl}$ ), plaquetas normales sin datos de hemólisis y consumo de complemento con descenso de C1q y C3, reactantes de fase aguda normales. El estudio de autoinmunidad reportó ANA positivo a título alto de 1/320 con patrón moteado fino, anti-DNA positivo (título de $57 \mathrm{Ud} / \mathrm{ml}$ ) con anti-Sm y anti-RNP positivos.

Se diagnosticó como nefropatía lúpica probable tipo $\mathrm{V}$ con debut en la gestación y se inició tratamiento con azatioprina a dosis de $1,5 \mathrm{mg} / \mathrm{kg} / \mathrm{dí}$.

A las $24+5$ SG se inició tratamiento con metilprednisolona en bolo durante 3 días ( $750 \mathrm{mg} / 24 \mathrm{~h}$ ), con posterior inicio de prednisona $50 \mathrm{mg}$ cada 24 horas e hidroxicloroquina $200 \mathrm{mg}$ cada 12 horas. Mantuvo un síndrome nefrótico sintomático, con posterior respuesta a albúmina, furosemida y antialdosterónicos (Fig. 2).

En los controles posteriores se evidenció la aparición de hematuria con dismorfia en varias determinaciones, sin HTA y sin deterioro de la función renal. En la $27+$ 4 SG se decidió el cambio de inmunosupresor de inducción por ciclofosfamida intravenosa según el esquema de Eurolupus $(500 \mathrm{mg} \times 6 \text { quincenal })^{13}$. En el seguimiento, mejora el síndrome nefrótico y aumenta la albúmina en cifras de 2,2 $\mathrm{g} / \mathrm{dl}$ en sangre.

En la 34 + 4 SG ingresó por rotura prematura de membranas, por lo que se indicó finalización electiva del parto mediante inducción médica. Se produjo un parto vaginal del que nació un varón vivo de 1598 gramos y Apgar 10/10.

El recién nacido tuvo una evolución favorable sin presentar complicaciones derivadas de la prematuridad ni lupus neonatal, y la paciente permanece estable a los 24 meses del parto.

\section{Método}

Se realizó una búsqueda bibliográfica entre los meses de mayo de 2020 y septiembre de 2020 en las bases de datos Web Of Science y Medline vía PubMed, con los términos "lupus nephritis gestation", "systemic lupus erythematosus", "lupus complications in pregnancy" y "lupus nephritis management", de revisiones sistemáticas, reportes y series de casos, y estudios observacionales publicados desde el año 1992 hasta junio de 2020 en inglés y español.

\section{Resultados}

Se encontraron 104 artículos, pero solo 52 mencionaban los términos "pregnancy" o "management", y 23 eran repetidos o no mencionaban el término "nephritis". Se seleccionaron 29 que trataban el tema en estudio y eran de interés para la revisión. Durante el proceso se revisaron también otros artículos que definen y actualizan los conceptos implicados en el manejo, el pronóstico y las complicaciones de la enfermedad tratada.

\section{Discusión}

El diagnóstico de brote lúpico durante el embarazo suele ser un desafío. Está bien establecido que las manifestaciones del brote pueden ser similares a las que el propio embarazo determina, como artralgias, fatiga o edemas. Para complicar aún más las cosas, las manifestaciones clínicas pueden confundirse 0 superponerse con las de la preeclampsia, pues ambas situaciones pueden presentarse con aumento de la proteinuria, deterioro de la función renal, hipertensión $y$ trombocitopenia.

En los casos clínicos que exponemos nos enfrentamos al reto de discernir entre brote de nefritis lúpica y preeclampsia con criterios de gravedad. A su vez, encontramos la dificultad añadida de diagnosticar un debut lúpico frente a una exacerbación de una enfermedad lúpica no diagnosticada (Tabla 3).

El diagnóstico diferencial entre ambas se ha visto mejorado con la reciente introducción en la práctica diaria de los marcadores angiogénicos de preeclampsia. No obstante, dado que solo conseguiríamos descartar una preeclampsia con un valor predictivo negativo del $98,5 \%$ y valores de ratio $<38^{14}$, cualquier valor superior a este no permitiría un diagnóstico diferencial fiable, por lo que, en el primer caso presentado, el diagnóstico tenía que ser clínico. El estudio de predicción de resultados a corto plazo en mujeres embarazadas con sospecha de preeclampsia (PROGNOSIS) evaluó si el cociente entre receptor soluble de tirosina cinasa y factor de crecimiento placentario (sFIt-1/PIGF) puede descartar preeclampsia en mujeres con sospecha de ella entre las 24 y 37 semanas de gestación. El estudio PROGNOSIS demostró que la relación sFIt-1/PIGF puede descartar la enfermedad durante 1 semana en mujeres que presentan un alto riesgo, con un valor predictivo negativo elevado ${ }^{15}$ (Tabla 4).

Por otra parte, las tasas de preeclampsia fueron más altas en las mujeres con nefropatía no diabética, lo que 

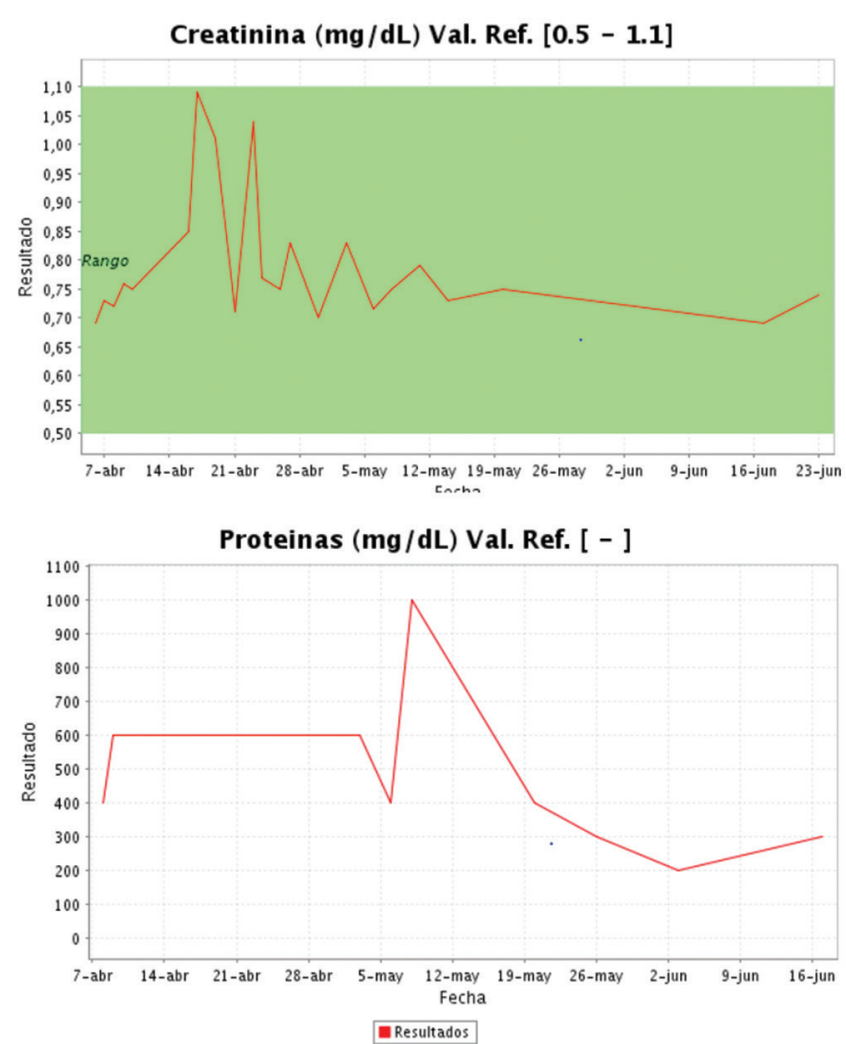

Constantes por Día

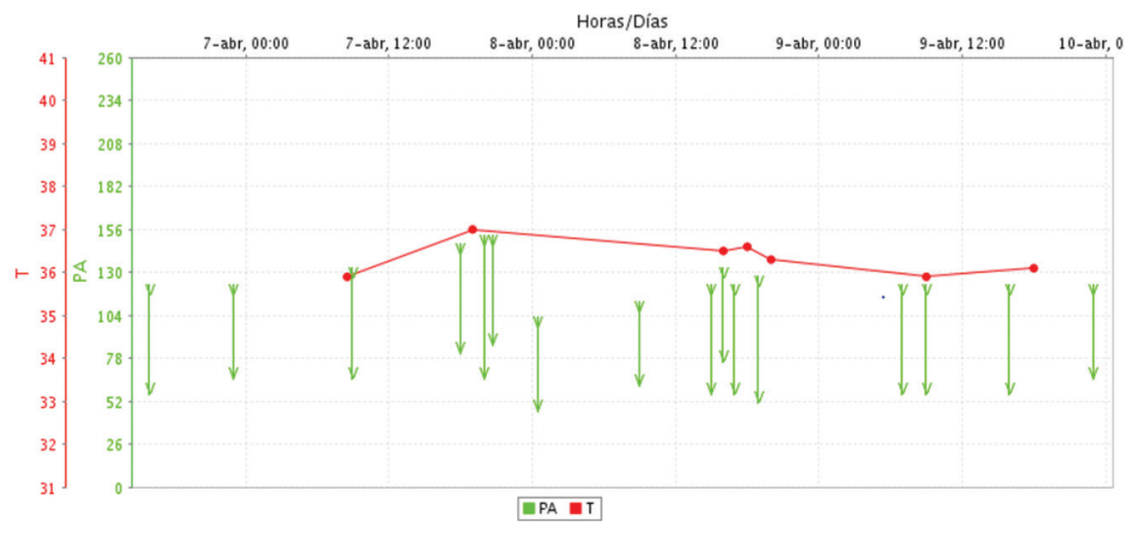

Figura 1. Evolución de las constantes del caso clínico 1.

FC: frecuencia cardíaca; PA: presión arterial; T: temperatura.

sugiere que la enfermedad glomerular podría ser particularmente peligrosa ${ }^{16}$. En un metaanálisis se reportó que la frecuencia de preeclampsia en pacientes con LES es del $7,6 \%$ y la de eclampsia es del $0,8 \%$. Asimismo, la presencia de afección renal activa en el momento del embarazo es un factor de riesgo para el desarrollo de preeclampsia, pero no de eclampsia ${ }^{17,18}$.

Una revisión sistemática publicada en 2017 por Blom et al..$^{19}$ halló que las mujeres con nefritis lúpica previa, a pesar de una tasa de filtrado glomerular preservada, tienen tasas más altas de parto prematuro y preeclampsia de inicio más temprano que las mujeres con lupus sin afectación renal. En la misma línea, el estudio publicado por Waness et al. ${ }^{20}$ informó un aumento de las probabilidades de parto prematuro de un $15 \%$ por cada aumento de la proteinuria de $1 \mathrm{~g} / \mathrm{dl}$ en cada trimestre.

El estudio de Carmona et al. ${ }^{21}$ sugirió que las mujeres con nefritis lúpica de clases III y IV tienen más probabilidades de embarazos complicados por 


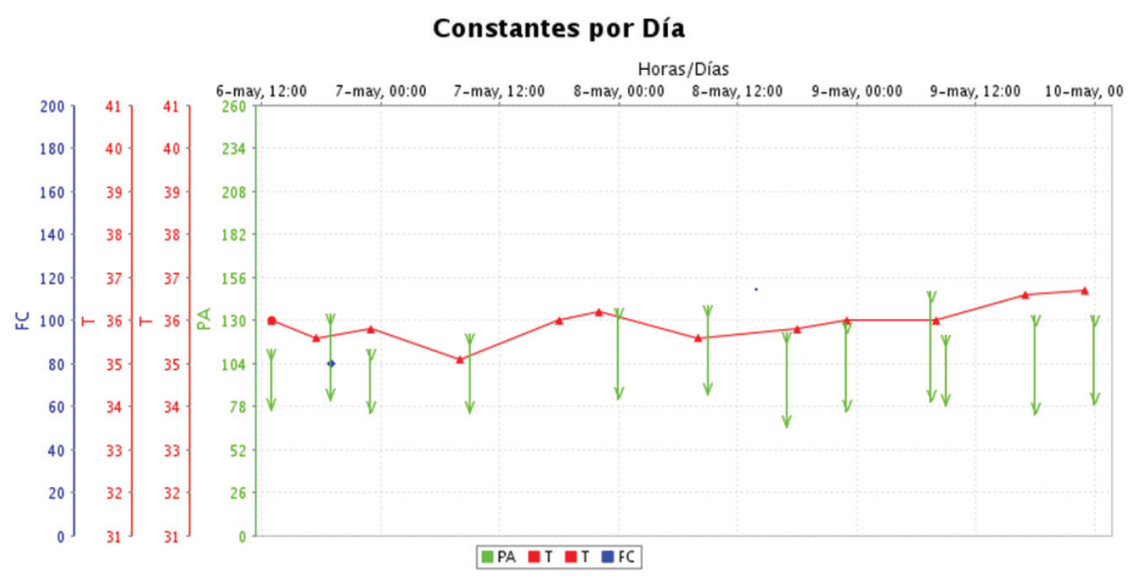

Figura 2. Evolución de presión arterial del caso clínico 1. FC: frecuencia cardíaca; PA: presión arterial; T: temperatura.

preeclampsia y CIR que aquellas con nefritis lúpica de las clase II y V, pero la clase histológica no se asoció con diferentes resultados en el metaanálisis antes mencionado?

Por otro lado, en ambos casos clínicos, aunque la balanza se inclinaba hacia el diagnóstico de debut de enfermedad lúpica con afectación renal, era necesario realizar un diagnóstico diferencial con una exacerbación de un lupus no diagnosticado. Esta posibilidad quedó descartada porque los datos de las historias clínicas no eran lo suficientemente sólidos como para apoyar esta hipótesis.

Otro recurso destacable en el diagnóstico diferencial de esta patología es la biopsia renal. En los casos reportados no se menciona la biopsia renal para apoyo del diagnóstico ya que no estaba suficientemente justificada su realización. Se sugiere realizar biopsia renal antes de las 32 SG en pacientes en las que no exista contraindicación, cuando la sospecha clínica no sea suficiente o no haya una respuesta adecuada al tratamiento ${ }^{22,23}$.

Parece que es importante el papel del complemento en el desarrollo de la placenta y el feto nor$\mathrm{mal}^{24,25}$. Los brotes de LES a menudo se asocian con niveles bajos de $\mathrm{C} 3$ y $\mathrm{C} 4$, y los valores normales de C3 y C4 se asocian con una reducción de los brotes de LES $^{26}$.

Si bien los niveles de C4 y C3 se pueden ver afectados por el propio embarazo. Las tasas de exacerbación renal y enfermedad de novo fueron significativamente más altas en aquellas con anti-DNA positivo y complemento bajo (7 de 80, 8,6\%) o complemento bajo solo (es decir, C3 bajo, C4 bajo o ambos; 5 de 42, 11,9\%) que en pacientes sin actividad serológica al inicio del estudio (4 de 176, 2,3\%; $p<0,01)^{27}$.

En cuanto al tratamiento inmunosupresor, la metilprednisolona pulsada y la plasmaféresis son opciones que pueden aplicarse para un efecto más rápido ${ }^{19,28,29}$.

Con respecto al tratamiento, la mayoría de los inmunosupresores son teratogénicos, pero contamos con la azatioprina y la ciclosporina como los fármacos más utilizados, dado que se demostró su seguridad durante el embarazo ${ }^{19}$. La hidroxicloroquina representa en la actualidad la primera opción terapéutica para el LES durante la gestación. Es segura para el embarazo y se ha demostrado que está asociada con un riesgo reducido de bloqueo cardíaco congénito en los recién nacidos ${ }^{30}$.

La azatioprina es con frecuencia el fármaco de elección para mantener la enfermedad en reposo durante el embarazo, y por tanto es uno de los fármacos de elección en la terapia de matenimiento ${ }^{19,31,32}$. En cuanto a la exposición a ciclofosfamida en el último trimestre, se ha relacionado con $\mathrm{CIR}$, supresión de la hematopoyesis y deterioro neurológico ${ }^{31}$. En el segundo caso clínico se justifica su uso por ser una manifestación potencialmente mortal no tratándose del primer trimestre. Según la guía EULAR/ACR y los resultados del Euro-Lupus Nephritis Trial (ELNT), es la terapia de elección en la inducción a la remisión frente a la combinación de azatioprina con tacrolimus ${ }^{13,33,34}$.

Otro fármaco inmunosupresor, del grupo de los denominados ahorradores, es el tacrolimus. En nuestro caso no se utilizó por la escasa evidencia de su uso para la inducción a la remisión en caso de enfermedad grave; sin embargo, es importante considerarlo en la 
Tabla 4. Diagnóstico diferencial entre preeclampsia y brote lúpico renal

\begin{tabular}{l|l|l}
\hline Hipertensión & Aumento & $\begin{array}{l}\text { Lupus eritematoso } \\
\text { sistémico }\end{array}$ \\
\hline $\begin{array}{l}\text { Proteinuria } \\
\text { Hematuria }\end{array}$ & Alta & Descenso \\
\hline Cilindros & No & Alta \\
\hline $\begin{array}{l}\text { Calciuria } \\
\text { Uricemia }\end{array}$ & Baja & Microhematuria (+) \\
\hline $\begin{array}{l}\text { Complemento } \\
\text { Anticuerpos } \\
\text { anti-DNA }\end{array}$ & Normal o bajo & Sí \\
\hline $\begin{array}{l}\text { Respuesta a } \\
\text { esteroides }\end{array}$ & No & Normal \\
\hline $\begin{array}{l}\text { Creatinina } \\
\text { Modificada de refs. } 6 \text { y } 18 .\end{array}$ & En aumento \\
\hline
\end{tabular}

terapia de mantenimiento dado su perfil de seguridad durante el embarazo. La evidencia actual indica que con tacrolimus no hay un aumento en la tasa de malformaciones congénitas ${ }^{13}$.

El empeoramiento de la proteinuria en el embarazo plantea un desafío diagnóstico y terapéutico importante. Aunque la nefritis lúpica y la vasculitis pueden debutar durante el embarazo, el efecto de este sobre las otras enfermedades glomerulares primarias no está claro. Actualmente no hay otros biomarcadores establecidos para su uso en la gestación. Aunque se han descrito el antirreceptor de fosfolipasa A2 de tipo M en la nefropatía membranosa ${ }^{20}$ y el receptor activador de plasminógeno de urocinasa soluble en la glomeruloesclerosis focal y segmentaria ${ }^{9}$, su uso en el embarazo requiere más estudios.

Siguiendo con el tratamiento, la utilización de diuréticos del grupo de las sulfonamidas, como la furosemida, ha demostrado que no son teratogénicos, pero su uso debe limitarse a edemas graves y manejo caso a caso, cuidando de no producir hipovolemia e hipoperfusión placentaria, con balance hidroelectrolítico diario ${ }^{22,23}$.

En cuanto a las complicaciones neonatales, las mujeres con anti-SSA presentan riesgo de desarrollo de bloqueo cardíaco fetal debido a la transferencia placentaria de inmunoglobulinas que conduce a fibroelastosis endocárdica, estimándose en un 5\% de casos, aunque solo se ha observado con títulos $\geq 50 \mathrm{U} / \mathrm{ml}^{21}$. Por ello, se recomienda el tratamiento con hidroxicloroquina en las gestantes con LES ${ }^{35,36}$.

En el primer caso expuesto, el debut fue posterior a la semana 24, por lo que, a pesar de tener títulos elevados de anticuerpos anti-SSA/RO, no presentó datos de bloqueo cardíaco. En las pacientes con anticuerpos anti-SSA/RO o anticuerpos anti-SSB/LA, o ambos, no existe evidencia sobre la pauta precisa de periodicidad para realizar la ecocardiografía fetal. Se recomienda iniciar los controles en la semana 16 con objeto de medir el intervalo auriculoventricular y detectar de forma precoz un bloqueo auriculoventricular o una afectación miocárdica (fibroelastosis endocárdica y miocardiopatía dilatada) ${ }^{37,38}$.

En cuanto al seguimiento obstétrico de las pacientes con nefritis lúpica no existe un claro consenso en cuanto a la periodicidad ${ }^{38}$, aunque se considera recomendable que desde el inicio del embarazo y hasta la semana 28 las revisiones médicas sean mensuales, acortando el intervalo a 2-3 semanas entre las semanas 28 y 36 , y posteriormente cada semana hasta el parto. Cada mes deberían solicitarse un perfil hematológico y bioquímico básico, sedimento de orina y cociente de proteínas-creatinina o proteinuria de 24 horas, al igual que concentraciones de antiDNA y de complemento C3-C4 y CH50 cada 3 meses, 0 antes en función de la evolución ${ }^{37}$.

Los casos clínicos expuestos ponen de relieve la necesidad de ampliar el uso hospitalario de los factores angiogénicos como técnica complementaria, en caso de que exista componente de glomerulonefritis de cualquier origen y sospecha de preeclampsia, así como la importancia de la valoración temprana de forma multidisciplinaria.

\section{Conclusiones}

El diagnóstico diferencial del debut de nefritis lúpica durante la gestación continúa siendo un desafío, a pesar de los avances en cuanto a marcadores angiogénicos. La valoración clínica constituye la piedra angular de este proceso diagnóstico y de sus implicaciones en cuanto a complicaciones del embarazo actuales y futuras. Es importante el seguimiento conjunto con otros especialistas teniendo en cuenta el grado de insuficiencia renal, la coexistencia de HTA, la aparición de preeclampsia y el CIR.

\section{Financiamiento}

Las autoras declaran no tener ningún financiamiento. 


\section{Conflicto de intereses}

Las autoras declaran no tener ningún conflicto de intereses.

\section{Responsabilidades éticas}

Protección de personas y animales. Las autoras declaran que para esta investigación no se han realizado experimentos en seres humanos ni en animales.

Confidencialidad de los datos. Las autoras declaran que han seguido los protocolos de su centro de trabajo sobre la publicación de datos de pacientes.

Derecho a la privacidad y consentimiento informado. Las autoras declaran que en este artículo no aparecen datos de pacientes

\section{Bibliografía}

1. Meyer O. Making pregnancy safer for patients with lupus. Joint Bone Spine. 2004;71:178-82.

2. Wu J, Ma J, Zhang WH, Di W. Management and outcomes of pregnancy with or without lupus nephritis: a systematic review and meta-analysis. Ther Clin Risk Manag. 2018;14:885-901.

3. Chen S, Sun X, Wu B, Lian X. Pregnancy in women with systemic lupus erythematosus: a retrospective study of 83 pregnancies at a single centre. Int J Environ Res Public Health. 2015;12:9876-88.

4. Zhao $\mathrm{C}$, Zhao J, Huang $\mathrm{Y}$, Wang $Z$, Wang $\mathrm{H}$, Zhang $\mathrm{H}$, et al. New-onset systemic lupus erythematosus during pregnancy. Clin Rheumatol. 2013;32:815-22.

5. Lateef A, Petri M. Systemic lupus erythematosus and pregnancy. Rheum Dis Clin North Am. 2017;43:215-26.

6. Khamashta MA. Systemic lupus erythematosus and pregnancy. Best Pract Res Clin Rheumatol. 2006;20:685-94.

7. Valero De Bernabé J, Soriano T, Albaladejo R, Juarranz M, Calle ME, Martínez D, et al. Risk factors for low birth weight: a review. Eur J Obstet Gynecol Reprod Biol. 2004;116:3-15.

7. Stanhope TJ, White WM, Moder KG, Smyth A, Garovic VD. Obstetric nephrology: lupus and lupus nephritis in pregnancy. Clin J Am Soc Nephrol. 2012;7:2089-99.

8. Magee LA, von Dadelszen P, Rey E, Ross S, Asztalos E, Murphy KE, et al. Less-tight versus tight control of hypertension in pregnancy. N Eng J Med. 2015;372:407-17.

9. Bramham K, Hunt BJ, Bewley S, Germain S, Calatayud I, Khamashta MA et al. Pregnancy outcomes in systemic lupus erythematosus with and without previous nephritis. J Rheumatol. 2011;38:1906-13.

10. Zhang JJ, Ma XX, Hao L, Liu LJ, Lv JC, Zhang H. A systematic review and meta-analysis of outcomes of pregnancy in CKD and CKD outcomes in pregnancy. Clin J Am Soc Nephrol. 2015;10:1964-78.

11. Bujold E, Roberge S, Lacasse Y, Bureau M, Audibert F, Marcoux S, et al. Prevention of preeclampsia and intrauterine growth restriction with aspirin started in early pregnancy: a meta-analysis. Obstet Gynecol. 2010;116:402-14.

12. Hofmeyr GJ, Roodt A, Atallah AN, Duley L. Calcium supplementation to prevent pre-eclampsia - a systematic review. S Afr Med J. 2003;93:224-8.

13. Houssiau FA, Vasconcelos C, D'Cruz D, Sebastiani GD, Garrido Ed Ede R, Danieli MG, et al. Immunosuppressive therapy in lupus nephritis: the Euro-Lupus Nephritis Trial, a randomized trial of low-dose versus high-dose intravenous cyclophosphamide. Arthritis Rheum. 2002;46:2121-31.

14. Agrawal S, Cerdeira AS, Redman C, Vatish M. Meta-analysis and systematic review to assess the role of soluble FMS-like tyrosine kinase- 1 and placenta growth factor ratio in prediction of preeclampsia: the SaPPPhirE study. Hypertension. 2018;71:306-16.

15. Verlohren S, Dröge LA. The diagnostic value of angiogenic and antiangiogenic factors in differential diagnosis of preeclampsia. Am J Obstet Gynecol. 2020 Sep 28:S0002-9378(20)31169-8. doi: 10.1016/j. ajog.2020.09.046. Online ahead of print.
16. De Jesús GR, De Jesús NR, Levy RA, Klumb EM. The use of angiogenic and antiangiogenic factors in the differential diagnosis of pre-eclampsia, antiphospholipid syndrome nephropathy and lupus nephritis. Lupus. 2014;23:1299-301.

17. Sibai BM. Imitators of severe pre-eclampsia. Semin Perinatol. 2009;33:196-205.

18. Barroso-Domínguez S. Tratamiento de la nefropatía lúpica en la embarazada. Ginecol Obstet Mex. 2018;86:478-84.

19. Blom K, Odutayo A, Bramham K, Hladunewich MA. Pregnancy and glomerular disease: a systematic review of the literature with management guidelines. Clin J Am Soc Nephrol. 2017;12:1862-72.

20. Waness A, Al Sayyari A, Salih SB, Al Shohaib S. Increased risk of hypertension, proteinuria and preeclampsia in pregnant Saudi females with IgA nephropathy. Hypertens Pregnancy. 2010;29:385-9.

21. Carmona F, Font J, Moga I, Làzaro I, Cervera R, Pac V, et al. Class IIIIV proliferative lupus nephritis and pregnancy: a study of 42 cases. Am J Reprod Immunol. 2005;53:182-8.

22. Gleeson S, Lightstone L. Glomerular disease and pregnancy. Adv Chronic Kidney Dis. 2020;27:469-76.

23. Hladunewich MA, et al. Pregnancy in women with underlying renal disease. En: UpToDate, Post TW (Ed), UpToDate, Hladunewich MA. (accedido el 1 de septiembre de 2020).. Disponible en: https://www.uptodate. com/contents/pregnancy-in-women-with-nondialysis-chronic-kidney-disease? search=Pregnancy $\% 20$ in $\% 20$ women $\% 20$ with $\% 20$ underlying $\% 20$ renal\%20disease\&source $=$ search_result\&selectedTitle=1 150\&usage type=default\&display_rank=1

24. Smyth A, Oliveira GH, Lahr BD, Bailey KR, Norby SM, Garovic VD. A systematic review and meta-analysis of pregnancy outcomes in patients with systemic lupus erythematosus and lupus nephritis. Clin J Am Soc Nephrol. 2010;5:2060-8.

25. Regal JF, Gilbert JS, Burwick RM. The complement system and adverse pregnancy outcomes. Mol Immunol. 2015;67:56-70.

26. Birmingham DJ, Irshaid F, Nagaraja HN, Zou X, Tsao BP, Wu H, et al. The complex nature of serum $\mathrm{C} 3$ and $\mathrm{C} 4$ as biomarkers of lupus renal flare. Lupus. 2010;19:1272-80.

27. Rodríguez Almaraz E, Sáez-Comet L, Casellas M, Delgado P, Ugarte A, Vela-Casasempere $P$, et al. Pregnancy control in patients with systemic lupus erytematosus/antiphospholipid syndrome. Reumatol Clin. 2021;17:125-31.

28. Moroni G, Doria A, Giglio E, Imbasciati E, Tani C, Zen M, et al. Maternal outcome in pregnant women with lupus nephritis. A prospective multicenter study. J Autoimmun. 2016;74:194-200.

29. Kattah AG, Garovic VD. Pregnancy and lupus nephritis. Semin Nephrol. 2015;35:487-99

30. Izmirly PM, Costedoat-Chalumeau N, Pisoni CN, Khamashta MA, Kim MY, Saxena A, et al. Maternal use of hydroxychloroquine is associated with a reduced risk of recurrent anti-SSA/Ro-antibody-associated cardiac manifestations of neonatal lupus. Circulation. 2012;126:76-82.

31. Coscia LA, Constantinescu S, Moritz MJ, Frank AM, Ramirez CB, Maley WR, et al. Report from the National Transplantation Pregnancy Registry (NTPR): outcomes of pregnancy after transplantation. Clin Transpl. 2010;65-85.

32. Zemlickis D, Lishner M, Degendorfer $P$, Panzarella $T$, Sutcliffe SB Koren G. Fetal outcome after in utero exposure to cancer chemotherapy. Arch Intern Med. 1992;152:573-6.

33. Götestam Skorpen C, Hoeltzenbein M, Tincani A, Fischer-Betz R Elefant E, Chambers $C$, et al. The EULAR points to consider for use of antirheumatic drugs before pregnancy, and during pregnancy and lactation. Ann Rheum Dis. 2016;75:795-810.

34. Aringer M. EULAR/ACR classification criteria for SLE. Semin Arthritis Rheum. 2019;49(3S):S14-S17.

35. Jaeggi E, Laskin C, Hamilton R, Kingdom J, Silverman E. The importance of the level of maternal anti-Ro/SSA antibodies as a prognostic marker of the development of cardiac neonatal lupus erythematosus a prospective study of 186 antibody-exposed fetuses and infants. J Am Coll Cardiol. 2010;55:2778-84.

36. Moroni G, Doria A, Giglio E, Tani C, Zen M, Strigini F, et al. Fetal outcome and recommendations of pregnancies in lupus nephritis in the $21^{\text {st }}$ century. A prospective multicenter study. J Autoimmun. 2016;74:6-12.

37. Moroni G, Quaglini S, Banfi G, Caloni M, Finazzi S, Ambroso G, et al. Pregnancy in lupus nephritis. Am J Kidney Dis. 2002;40:713-20.

38. Irene T, Narcís M, Martínez JM, Ramírez JC. Protocolo sobre lupus eritematoso sistémico y embarazo. Servicio de enfermedades autoinmunes, Institut Clinic de Medicina i Dermatología: Emmanuel Coloma, Carolina Donate, Gerad Espinosa. Febrero 2013. Disponible en: https:// medicinafetalbarcelona. org/protocolos/es/patologia-maternaobstetrica/ les $\% 20 y \% 20$ embarazo.pdf 